\title{
MULTIDIMENSIONAL DECAY IN VAN DER CORPUT LEMMA
}

\author{
MICHAEL RUZHANSKY
}

\begin{abstract}
In this paper we present a multidimensional version of the van der Corput lemma where the decay of the oscillatory integral is gained with respect to all space variables, connecting the standard one-dimensional van der Corput lemma with the stationary phase method.
\end{abstract}

\section{INTRODUCTION}

This paper is devoted to the estimates for the oscillatory integrals of the type

$$
I(\lambda)=\int_{\mathbb{R}^{N}} e^{i \lambda \Phi(x)} a(x) d x,
$$

where the support of $a \in C_{0}^{\infty}\left(\mathbb{R}^{N}\right)$ is sufficiently small. The estimate for $I(\lambda)$ as $\lambda \rightarrow \infty$ is well-known in one dimension $N=1$. If $\Phi$ is real-valued and $\left|\Phi^{(k)}(x)\right| \geq 1$ on the support of $a$, then the estimate $|I(\lambda)| \leq c_{k} \lambda^{-1 / k}$ holds when $k \geq 2$, or when $k=1$ and $\Phi^{\prime}(x)$ is monotonic. In this case the bound $c_{k}$ is also independent of $\Phi$ and $\lambda$ (see e.g. Sogge [10] or Stein [11]), and the decay rate is sharp. This result plays a crucial role in various areas of analysis. For example, it is closely related to sublevel set estimates of the form

$$
\text { meas }\{s \in \operatorname{supp} a:|\Phi(s)| \leq t\} \leq c_{k} t^{1 / k},
$$

where $\Phi$ is a function as above, with numerous applications in partial differential equations, microlocal analysis, harmonic analysis, etc.

A multidimensional version of these results would be of great value, but presents many difficulties. It is known that for dimensions $N \geq 1$, if, for example, $\partial^{\alpha} \Phi \geq 1$ on $\operatorname{supp} a$, then $|I(\lambda)| \leq c_{\alpha}|\lambda|^{-1 /|\alpha|}$. The decay rate here is sharp, but the constant $c_{\alpha}$ may depend on $\Phi$ and the estimate does not scale well. Again, such estimate is closely related to the multilinear sublevel set problem (see e.g. Phong, Stein and Sturm [7]). Parameter dependent sublevel set estimates were recently used by Kamotski and Ruzhansky [5] in the analysis of elliptic and hyperbolic systems with multiplicities, to yield Sobolev space estimates for relevant classes of oscillatory integrals and for the solutions.

Recently, Christ, Carbery and Wright [2] and Carbery and Wright [3], proposed versions of van der Corput lemma for functions of several variables, in formulations where the constant in the estimate is independent of the phase function. This aspect is of significant importance for applications allowing to investigate various perturbation and other properties of appearing integrals. However, the decay rate of the

Date: October 30, 2018.

The author was supported by the Leverhulme Research Fellowship and by EPSRC grant $\mathrm{EP} / \mathrm{E} 062873 / 01$. 
corresponding oscillatory integral there is again essentially one-dimensional because the non-degeneracy of only one (higher-order) derivative is assumed.

At the same time, the decay rate exhibited in many problems of interest is better than one-dimensional. If one compares this with the case of non-degenerate stationary points of $\Phi$, the stationary phase method will readily yield the decay rate $|I(\lambda)| \leq$ $C \lambda^{-N / 2}$. However, if a stationary point degenerates, the situation becomes much more delicate (see e.g. Hörmander [4, Chapter 7]), and no good estimates are available in general.

The aim of the present paper is to bridge the gap between van der Corput lemma and estimates provided by the stationary phase method. On one hand, the standard van der Corput lemma works well for degeneracies of high orders but produces only one-dimensional decay rate. On the other hand, the stationary phase method produces the multidimensional decay rate, but does not work well for degenerate stationary points.

The result of this paper will yield a multidimensional decay rate for degenerate stationary points. We will identify a class of functions, for which this can be achieved. These functions will have certain convexity type properties. It is clear that certain convexity conditions are necessary to ensure the multidimensional decay rate. In fact, conditions of the one-dimensional van der Corput lemma guarantee that the function (or some derivative of the function) is convex in one dimension. Thus, it is natural that an analogue of convexity also appears in several dimensions to ensure that we gain one-dimensional decays in all directions. It is then a question of putting all these rates together to yield the full multidimensional decay, which will turn out to be $N$-times better than the standard van der Corput estimate.

In what follows we will also allow phase function $\Phi$ to be complex valued and to depend on an arbitrary set of parameters. These two situations often happen in applications to partial differential equations, in particular in the analysis of solutions represented as oscillatory integrals, leading to the dispersive and to the subsequent Strichartz estimates. Thus, the complex phase corresponds to the fact that characteristics of the analysed evolution equations may be complex (see e.g. Trèves [13]). At the same time, the dependence of the phase and of the amplitude on parameters is also essential, and is related to uniform estimates of [2]. Also, in applications to the Strichartz estimates for hyperbolic equations of high orders considered by Ruzhansky and Smith [9], a parameter is essential to encode the information on low order perturbations of the equation, in order to establish the dispersive estimates for solutions uniformly over such perturbations. At the same time, in hyperbolic equations with time dependent coefficients (e.g. considered by Matsuyama and Ruzhansky [6]), the parameter encodes the information on the perturbations of the limiting behaviour of coefficients, again allowing to obtain dispersive estimates uniformly over such perturbations. We will leave out these and other applications outside the scope of this short paper.

We will use the standard multi-index notation $\alpha=\left(\alpha_{1}, \ldots, \alpha_{N}\right)$, denote its length by $|\alpha|=\alpha_{1}+\cdots+\alpha_{N}$ and partial derivatives by $\partial^{\alpha}=\partial_{x_{1}}^{\alpha_{1}} \cdots \partial_{x_{N}}^{\alpha_{N}}$. We will also use the standard convention to denote all constants by letter $C$ although they may have different values on different occasions. 


\section{Multidimentional van Der Corput lemma}

The following theorem is the main result that establishes the multidimensional decay rate for a class of oscillatory integrals.

Theorem 2.1. Consider the oscillatory integral

$$
I(\lambda, \nu)=\int_{\mathbb{R}^{N}} e^{i \lambda \Phi(x, \nu)} a(x, \nu) \chi(x) d x,
$$

where $N \geq 1$, and $\nu$ is a parameter. Let $\gamma \geq 2$ be an integer. Assume that

(A1) there exists a sufficiently small $\delta>0$ such that $\chi \in C_{0}^{\infty}\left(B_{\delta / 2}(0)\right)$, where $B_{\delta / 2}(0)$ is the ball with radius $\delta / 2$ around 0 ;

(A2) $\Phi(x, \nu)$ is a complex valued function such that $\operatorname{Im} \Phi(x, \nu) \geq 0$ for all $x \in$ supp $\chi$ and all parameters $\nu$;

(A3) for some fixed $z \in \operatorname{supp} \chi$, the function

$$
F(\rho, \omega, \nu):=\Phi(z+\rho \omega, \nu),|\omega|=1,
$$

satisfies the following conditions. Assume that for each $\mu=(\omega, \nu)$, function $F(\cdot, \mu)$ is of class $C^{\gamma+1}$ on $\operatorname{supp} \chi$, and let us write its $\gamma^{\text {th }}$ order Taylor expansion in $\rho$ at 0 as

$$
F(\rho, \mu)=\sum_{j=0}^{\gamma} a_{j}(\mu) \rho^{j}+R_{\gamma+1}(\rho, \mu),
$$

where $R_{\gamma+1}$ is the remainder term. Assume that we have

(F1) $a_{0}(\mu)=a_{1}(\mu)=0$ for all $\mu$;

(F2) there exists a constant $C>0$ such that $\sum_{j=2}^{\gamma}\left|a_{j}(\mu)\right| \geq C$ for all $\mu$;

(F3) for each $\mu,\left|\partial_{\rho} F(\rho, \mu)\right|$ is increasing in $\rho$ for $0<\rho<\delta$;

(F4) for each $k \leq \gamma+1, \partial_{\rho}^{k} F(\rho, \mu)$ is bounded uniformly in $0<\rho<\delta$ and $\mu$;

(A4) for each multi-index $\alpha$ of length $|\alpha| \leq\left[\frac{N}{\gamma}\right]+1$, there exists a constant $C_{\alpha}>0$ such that $\left|\partial_{x}^{\alpha} a(x, \nu)\right| \leq C_{\alpha}$ for all $x \in \operatorname{supp} \chi$ and all parameters $\nu$.

Then there exists a constant $C=C_{N, \gamma}>0$ such that

$$
|I(\lambda, \nu)| \leq C(1+\lambda)^{-\frac{N}{\gamma}} \quad \text { for all } \lambda \in[0, \infty) \text { and all parameters } \nu .
$$

Theorem 2.1 obviously includes the case where $a$ and $\Phi$ depend on different sets of parameters. In this case we may let $\nu$ run over the whole space of parameters.

We also note that assumption (A3), or rather (F3), can be view as an analogue of a convexity assumption. Indeed, if $F$ is real valued, then (F3) implies that the second order derivative $\partial_{\rho}^{2} F(\rho, \mu)$ does not change sign for $0<\rho<\delta$, because $\partial_{\rho} F(0, \mu)=0$ by (F1). In turn, condition (F1) is not restrictive, since $a_{0}(\mu)$ can be taken out of the integral, and non-zero $a_{1}(\mu)$ would actually give a faster decay rate.

Proof. It is clear that (2.1) holds for $0 \leq \lambda \leq 1$ since $|I(\lambda, \nu)|$ is bounded for such $\lambda$, in view of assumptions (A1), (A2) and (A4), So, we may consider the case where $\lambda \geq 1$. Let $z \in \mathbb{R}^{N}$ be as in (A3), and set $x=z+\rho \omega$, where $\omega \in \mathbb{S}^{N-1}, \rho>0$. For $N=1$, we use $\mathbb{S}^{0}=\{-1,1\}$. Then we can write

$$
I(\lambda, \nu)=\int_{\mathbb{S}^{N-1}} \int_{0}^{\infty} e^{i \lambda \Phi(z+\rho \omega, \nu)} a(z+\rho \omega, \nu) \chi(z+\rho \omega) \rho^{N-1} d \rho d \omega .
$$


It suffices to prove (2.1) for the inner integral.

Choose a function $\theta \in C_{0}^{\infty}([0, \infty)), 0 \leq \theta(s) \leq 1$ for all $s$, such that $\theta(s)$ is identically 1 for $0 \leq s \leq \frac{1}{2}$ and is identically zero for $s \geq 1$. Then with our notation $F(\rho, \omega, \nu)=\Phi(z+\rho \omega, \nu)$, we split the inner integral into the sum of the two integrals

$$
\begin{gathered}
I_{1}(\lambda, \nu, \omega, z)=\int_{0}^{\infty} e^{i \lambda F(\rho, \omega, \nu)} a(z+\rho \omega, \nu) \chi(z+\rho \omega) \theta\left(\lambda^{\frac{1}{\gamma}} \rho\right) \rho^{N-1} d \rho, \\
I_{2}(\lambda, \nu, \omega, z)=\int_{0}^{\infty} e^{i \lambda F(\rho, \omega, \nu)} a(z+\rho \omega, \nu) \chi(z+\rho \omega)(1-\theta)\left(\lambda^{\frac{1}{\gamma}} \rho\right) \rho^{N-1} d \rho .
\end{gathered}
$$

Let us first estimate $I_{1}=I_{1}(\lambda, \nu, \omega, z)$. Since $\theta\left(\lambda^{\frac{1}{\gamma}} \rho\right)=0$ for $\lambda^{\frac{1}{\gamma}} \rho \geq 1$, changing variable $\tau=\lambda^{\frac{1}{\gamma}} \rho$, we have

$$
\left|I_{1}\right| \leq C \int_{0}^{\infty} \theta\left(\lambda^{\frac{1}{\gamma}} \rho\right) \rho^{N-1} d \rho=C \int_{0}^{\infty} \tau^{N-1} \lambda^{-\frac{N-1}{\gamma}} \theta(\tau) \lambda^{-\frac{1}{\gamma}} d \tau,
$$

which yields the following estimate for $I_{1}$ :

$$
\left|I_{1}\right| \leq C \lambda^{-\frac{N}{\gamma}} \int_{0}^{1} \tau^{N-1} d \tau \leq C \lambda^{-\frac{N}{\gamma}}
$$

In order to estimate $I_{2}=I_{2}(\lambda, \nu, \omega, z)$, let us first establish a useful estimate for functions $F$ satisfying condition (F3). We claim that under condition (A3), or rather under (F1) (F4), there exist constants $C, C_{m}>0$ such that we have estimates

$$
\begin{gathered}
\left|\partial_{\rho} F(\rho, \mu)\right| \geq C \rho^{\gamma-1} \\
\text { and }\left|\partial_{\rho}^{m} F(\rho, \mu)\right| \leq C_{m} \rho^{1-m}\left|\partial_{\rho} F(\rho, \mu)\right|,
\end{gathered}
$$

for all $0<\rho<\delta$, all parameters $\mu$, and all $m \leq \gamma+1$. First, note that for $0<\rho \leq 1$ and $m=\gamma+1$, estimate (2.4) follows from (2.3) and assumption (F4). So we may only consider $m \leq \gamma$.

Now, assumption (F2) implies that

$$
\pi(\rho, \mu):=\sum_{j=2}^{\gamma} j\left|a_{j}(\mu)\right| \rho^{j-1} \geq C \rho^{\gamma-1} .
$$

Thus, in order to prove (2.3), it suffices to show that

$$
\left|\partial_{\rho} F(\rho, \mu)\right| \geq C \pi(\rho, \mu) \text { for all } 0<\rho<\delta \text { and all parameters } \mu \text {. }
$$

For $1 \leq m \leq \gamma$, we have, using (A3),

$$
\partial_{\rho}^{m} F(\rho, \mu)=\sum_{k=0}^{\gamma-m} \frac{(k+m) !}{k !} a_{k+m}(\mu) \rho^{k}+R_{m, \gamma-m}(\rho, \mu),
$$

where $R_{m, \gamma-m}(\rho, \mu)=\int_{0}^{\rho} \partial_{s}^{\gamma+1} F(s, \mu) \frac{(\rho-s)^{\gamma-m}}{(\gamma-m) !} d s$ is the remainder term of the $(\gamma-m)^{\text {th }}$ Taylor expansion of $\partial_{\rho}^{m} F(\rho, \mu)$. By (F4) and (2.5), we get that

$$
\left|R_{m, \gamma-m}(\rho, \mu)\right| \leq C_{\gamma, m} \rho^{\gamma-m+1} \leq C_{\gamma, m} \pi(\rho, \mu) \rho^{2-m} \quad \text { for } 0<\rho<\delta .
$$


Hence, for $0<\rho<\delta$, we have

$$
\begin{aligned}
\left|\partial_{\rho} F(\rho, \mu)\right| & =\left|\sum_{k=0}^{\gamma-1}(k+1) a_{k+1}(\mu) \rho^{k}+R_{1, \gamma-1}(\rho, \mu)\right| \\
& \geq\left|\sum_{j=2}^{\gamma} j a_{j}(\mu) \rho^{j-1}\right|-\left|R_{1, \gamma-1}(\rho, \mu)\right| \geq\left|\sum_{j=2}^{\gamma} j a_{j}(\mu) \rho^{j-1}\right|-C_{\gamma} \pi(\rho, \mu) \rho .
\end{aligned}
$$

It follows now from assumptions (F1) and (F3) that

$$
\begin{aligned}
&\left|\partial_{\rho} F(\rho, \mu)\right|=\max _{0 \leq \sigma \leq \rho}\left|\partial_{\rho} F(\sigma, \mu)\right| \\
& \geq \max _{0 \leq \sigma \leq \rho}\left|\sum_{j=2}^{\gamma} j a_{j}(\mu) \sigma^{j-1}\right|-\max _{0 \leq \sigma \leq \rho} C_{\gamma} \pi(\sigma, \mu) \sigma \\
&=\max _{0 \leq \bar{\sigma} \leq 1}\left|\sum_{j=2}^{\gamma} j a_{j}(\mu) \rho^{j-1} \bar{\sigma}^{j-1}\right|-C_{\gamma} \pi(\rho, \mu) \rho,
\end{aligned}
$$

since $\pi(\sigma, \mu) \sigma=\sum_{j=2}^{\gamma} j\left|a_{j}(\mu)\right| \sigma^{j}$ achieves its maximum on $0 \leq \sigma \leq \rho$ at $\sigma=\rho$. Noting that

$$
\max _{0 \leq \bar{\sigma} \leq 1}\left|\sum_{j=2}^{\gamma} z_{j} \bar{\sigma}^{j-1}\right| \quad \text { and } \quad \sum_{j=2}^{\gamma}\left|z_{j}\right|
$$

are both norms on $\mathbb{C}^{\gamma-1}$ and, hence, are equivalent, we immediately get

$$
\begin{aligned}
\left|\partial_{\rho} F(\rho, \mu)\right| & \geq C \sum_{j=2}^{\gamma} j\left|a_{j}(\mu)\right| \rho^{j-1}-C_{\gamma} \pi(\rho, \mu) \rho \\
& \geq\left(C-C_{\gamma} \delta\right) \pi(\rho, \mu) \geq C \pi(\rho, \mu),
\end{aligned}
$$

for some constants $C>0$, if $\delta$ is sufficiently small. This completes the proof of (2.6).

To prove (2.4), we will use the representation (2.7). Since $1 \leq m \leq \gamma$, it follows from the definition of $\pi(\rho, \mu)$ that

$$
\left|\sum_{k=0}^{\gamma-m} \frac{(k+m) !}{k !} a_{k+m}(\mu) \rho^{k}\right| \leq C_{m} \pi(\rho, \mu) \rho^{1-m},
$$

which, together with (2.8) and (2.6), yields

$$
\left|\partial_{\rho}^{m} F(\rho, \mu)\right| \leq C_{m, \delta} \rho^{1-m}\left|\partial_{\rho} F(\rho, \mu)\right| \text { for } 0<\rho<\delta .
$$

This completes the proof of the claimed estimates (2.3) and (2.4).

Let us now come back to the estimate for $I_{2}$. Define the operator

$$
L:=\left(i \lambda \partial_{\rho} F(\rho, \omega, \nu)\right)^{-1} \frac{\partial}{\partial \rho}
$$

which clearly satisfies the useful identity $L\left(e^{i \lambda F(\rho, \omega, \nu)}\right)=e^{i \lambda F(\rho, \omega, \nu)}$. Denoting the adjoint of $L$ by $L^{*}$, we have, for each $l \in \mathbb{N} \cup\{0\}$,

$$
I_{2}=\int_{0}^{\infty} e^{i \lambda F(\rho, \omega, \nu)}\left(L^{*}\right)^{l}\left[a(z+\rho \omega, \nu) \chi(z+\rho \omega)(1-\theta)\left(\lambda^{\frac{1}{\gamma}} \rho\right) \rho^{N-1}\right] d \rho .
$$


Now,

$$
\left(L^{*}\right)^{l}=\left(\frac{i}{\lambda}\right)^{l} \sum C_{s_{1}, \ldots, s_{p}, p, r, l} \frac{\partial_{\rho}^{s_{1}} F \cdots \partial_{\rho}^{s_{p}} F}{\left(\partial_{\rho} F\right)^{l+p}}(\rho, \omega, \nu) \frac{\partial^{r}}{\partial \rho^{r}},
$$

where the sum is over all integers $s_{1}, \ldots, s_{p}, p, r \geq 0$ such that $s_{1}+\cdots+s_{p}+r-p=l$. From (2.3) and (2.4) it follows that

$$
\left|\frac{\partial_{\rho}^{s_{1}} F \ldots \partial_{\rho}^{s_{p}} F}{\left(\partial_{\rho} F\right)^{l+p}}(\rho, \omega, \nu)\right| \leq C \rho^{p-s_{1}-\cdots-s_{p}-l \gamma+l}=C \rho^{r-l \gamma} .
$$

Also, it is easy to see that for $r \leq\left[\frac{N}{\gamma}\right]+1$, we have

$$
\left|\frac{\partial^{r}}{\partial \rho^{r}}\left[a(z+\rho \omega, \nu) \chi(z+\rho \omega)(1-\theta)\left(\lambda^{\frac{1}{\gamma}} \rho\right) \rho^{N-1}\right]\right| \leq C_{N} \rho^{N-1-r} \widetilde{\chi}(\lambda, \rho),
$$

where $\tilde{\chi}(\lambda, \rho)$ is a smooth function in $\rho$ which is zero for $\lambda^{\frac{1}{\gamma}} \rho<\frac{1}{2}$. Let us now take $l=\left[\frac{N}{\gamma}\right]+1$, so that $N-l \gamma<0$. Then we can estimate

$$
\begin{aligned}
\left|I_{2}\right| & \leq C_{N} \lambda^{-l} \int_{0}^{\infty} \sum C_{s_{1}, \ldots, s_{p}, p, r, l} \rho^{r-l \gamma} \rho^{N-1-r} \tilde{\chi}(\lambda, \rho) d \rho \\
& \leq C_{N} \lambda^{-l} \int_{\frac{1}{2} \lambda^{-\frac{1}{\gamma}}}^{\infty} \rho^{N-1-l \gamma} d \rho=C_{N} \lambda^{-l}\left[\frac{\rho^{N-l \gamma}}{N-l \gamma}\right]_{\frac{1}{2} \lambda^{-\frac{1}{\gamma}}}^{\infty}=C_{N, \gamma} \lambda^{-\frac{N}{\gamma}} .
\end{aligned}
$$

Combining this estimate with estimate (2.2) for $I_{1}$, we obtain the desired estimate (2.1). This completes the proof of the theorem.

We note that in the proof we showed that if function $F$ satisfies conditions (F1) (F4), it also satisfies estimates (2.3) and (2.4). A version of this part of the argument was discussed by Sugimoto [12] for real valued analytic functions without dependence on $\mu$, where the analysis was based on the Cauchy's integral formula for analytic functions (see also Randol [8] and Beals [1]). The proof that we give for (2.3) and (2.4) extends it to the generality required for Theorem 2.1.

In fact, let us also briefly indicate a smooth version of these estimates. Suppose that a function $F(\cdot, \mu)$ is smooth in the first variable, and that it satisfies conditions (F1) (F3), as well as condition (F4) for all $m \in \mathbb{N}$. Then we claim that for sufficiently small $\delta>0$, estimates (2.3) and (2.4) are satisfied also for all $m \in \mathbb{N}$.

Indeed, we already proved estimate (2.3) and we also proved (2.4) for $m \leq \gamma$. It remains to consider the case $m>\gamma$. Since $\gamma+1-m \leq 0$, from (F4) it trivially follows that for $0<\rho<\delta$ we have a stronger estimate

$$
\left|\partial_{\rho}^{m} F(\rho, \mu)\right| \leq C_{m} \leq C_{m, \delta} \rho^{\gamma+1-m} \leq C_{m, \delta} \rho^{2-m}\left|\partial_{\rho} F(\rho, \mu)\right|,
$$

where the last inequality is a consequence of (2.3) .

\section{REFERENCES}

[1] R. M. Beals, $L^{p}$ boundedness of Fourier integral operators, Mem. Amer. Math. Soc. 38 (1982), no. 264.

[2] A. Carbery, M. Christ and J. Wright, Multidimensional van der Corput and sublevel set estimates, J. Amer. Math. Soc. 12 (1999), 981-1015.

[3] A. Carbery and J. Wright, What is van der Corput's lemma in higher dimensions?, Proceedings of the 6th International Conference on Harmonic Analysis and Partial Differential Equations (El Escorial, 2000). Publ. Mat. 2002, Vol. Extra, 13-26. 
[4] L. Hörmander, The analysis of linear partial differential operators. I, Springer-Verlag, Berlin, 1983.

[5] I. Kamotski and M. Ruzhansky, Regularity properties, representation of solutions and spectral asymptotics of systems with multiplicities, Comm. Partial Differential Equations, 32 (2007), $1-35$.

[6] T. Matsuyama and M. Ruzhansky, Asymptotic integration and dispersion for hyperbolic equations, with applications to Kirchhoff equations, preprint.

[7] D. H. Phong, E. M. Stein and J. Sturm, Multilinear level set operators, oscillatory integral operators, and Newton polyhedra, Math. Ann. 319 (2001), 573-596.

[8] B. Randol, On the asymptotic behavior of the Fourier transform of the indicator function of a convex set, Trans. Amer. Math. Soc. 139 (1969), 279-285.

[9] M. Ruzhansky and J. Smith, Dispersive and Strichartz estimates for hyperbolic equations with constant coefficients, preprint.

[10] C. D. Sogge, Fourier integrals in classical analysis, Cambridge Tracts in Mathematics, vol. 105, Cambridge University Press, Cambridge, 1993.

[11] E. M. Stein, Harmonic analysis: real-variable methods, orthogonality, and oscillatory integrals, Princeton Mathematical Series, vol. 43, Princeton University Press, Princeton, NJ, 1993.

[12] M. Sugimoto, A priori estimates for higher order hyperbolic equations, Math. Z. 215 (1994), 519-531.

[13] F. Trèves, Introduction to Pseudo-Differential and Fourier Integral Operators, Vol. II, Plenum Press, 1982.

Department of Mathematics

IMPERIAL COLLEGE LONDON

180 QueEn's GATE

LONDON SW7 2AZ

United KingDOM

E-mail address: m.ruzhansky@imperial.ac.uk 